\title{
The management of Sjögren's syndrome: British Society for Rheumatology guideline scope
}

Authorship list:

Elizabeth Price (corresponding author) - Great Western Hospital, Rheumatology, Marlborough Road, Swindon, SN36BB Elizabeth.price5@nhs.net

Alexander Allen - British Society for Rheumatology, London, UK

Saaeha Rauz - University of Birmingham, Academic Unit of Ophthalmology, Birmingham and Midland, Eye Centre, Dudley Road, Birmingham, B18 7QH

Anwar Tappuni - Queen Mary University of London, Institute of dentistry, Turner street, London, England, UK E1 2AD

Nurhan Sutcliffe - Barts and The London School of Medicine and Dentistry, Queen Mary University of London, Department of Rheumatology, Mile End Hospital, Bancroft Road, London, UK E1 4DG Michele Bombardieri - William Harvey Research Institute, Centre for Experimental Medicine and Rheumatology, London, UK

Carty, Sara - Great Western Hospital, Rheumatology, Swindon, UK

Coziana Ciurtin - University College London Hospitals NHS Trust, Department of Rheumatology, 3rd Floor Central, 250 Euston Road, London, UK NW1 2PG

Bridget Crampton - British Sjogren's Syndrome Association, British Sjogren's syndrome, Birmingham, UK

Lisa Duncalfe - New Queen Elizabeth Hospital Birmingham, Rheumatology, Birmingham, UK Benjamin Fisher - University of Birmingham, Rheumatology research Group, Centre for Translational Inflammation Research, University of Birmingham Research Laboratories, Queen Elizabeth Hospital, Birmingham, UK B15 2WB

Peter Glennon - NHS Shetland, Hub GP, Lerwick, Shetland, UK

Katie L. Hackett, - Northumbria University, Social Work, Education and Community Wellbeing Newcastle upon Tyne, Tyne and Wear, UK

Genevieve Larkin - King's College Hospital NHS Foundation Trust, Ophthalmology, London, UK Fai Ng: Newcastle University, Institute of Cellular Medicine, Newcastle upon Tyne, Tyne and Wear, UK

Athimalaipet V. Ramanan - University Hospitals Bristol and Weston NHS Foundation Trust, Paediatric rheumatology, Bristol, Bristol, UK

Saad Rassam- Maidstone and Tunbridge Wells NHS Trust, Haemato-Oncology, Maidstone, Kent, UK 
Stephen B Walsh - University College London, Centre for Nephrology, UCL Hampstead Campus, Royal Free Hospital, London, UK NW3 2PF

Simon Bowman - University Hospital Birmingham, Rheumatology Department, Selly Oak Hospital, Birmingham, UK B29 6JD

\section{Declarations of interests:}

Simon Bowman: Honoraria for consultancy with Novartis and Galapagos Pharmaceuticals Benjamin Fisher: has undertaken consultancy for Novartis, Roche, BMS, Servier, Galapagos and Janssen.

No other authors have financial conflicts of interest to declare

\section{Funding: this work was supported by the British Society for Rheumatology}




\section{The management of Sjögren's syndrome: British Society for Rheumatology guideline scope}

\section{Abstract:}

The guideline will be developed using the methods and processes outlined in Creating Clinical Guidelines: Our Protocol (1). This development process to produce guidance, advice and recommendations for practice has National Institute for Health and Care Excellence (NICE) accreditation.

\section{Introduction}

\section{Why the guideline is needed}

Health care workers often under-estimate the burden of Sjögren's syndrome (SS) and people with SS struggle with the chronicity of the condition and are frustrated by the paucity of treatment and lack of knowledge in the medical world. This will build on previous guidelines and continue to address these issues.

The most recent BSR guidelines on SS were published in 2017 (2). Since then the monitoring guidelines on hydroxychloroquine have changed, new eye drops have come to the market and research into the underlying mechanisms of disease has progressed with new treatments being trialled.

There are existing international treatment recommendations that may be applicable in the UK including those published by the European League Against Rheumatism (EULAR) in 2020 (3) and the North American guidelines published in 2016 (4). However, these are generic to many international health settings and do not provide specific guidance for the UK nor do they include the management of children. This guideline will aim to address management in both adults and children and will focus specifically on the healthcare setting in the UK. In doing so it will take into account issues specific to the UK based population the majority of whom will access healthcare through the National Health Service (NHS). It will advise on best practice standard of care within the UK and cover the patient journey from initial presentation to diagnosis and long term management taking into account specifics such as UK access to drugs.

\section{Key facts and figures}

SS is a chronic, auto-immune disease of unknown aetiology for which there is no known curative treatment. Successful management requires the physician to personalise the care to the individual. Although dryness (sicca) of the eyes and mouth are the classically described features, dryness of other mucosal surfaces and systemic manifestations, including fatigue and arthralgia, are common. 
Systemic features affect at least $70 \%$ and include inflammatory arthritis, skin involvement, haematological abnormalities, neuropathies, interstitial lung disease and a 5-10\% lifetime risk of Bcell lymphoma $(5,6)$. Cryoglobulinaemic vasculitis may affect $3.5-6.5 \%$ of SS patients and often presents as purpura early in the course of the disease. It predicts a more severe clinical course and higher risk of lymphoma development $(7,8)$. The physician should aim to empower the individual to manage their condition; conserving replacing and stimulating secretions; preventing damage and suppressing underlying systemic disease activity.

SS has a significant Impact on the quality of life (QoL) of affected patients. A recent literature review found that health-related (HR) QoL was markedly reduced in SS in multiple studies across many countries when compared with healthy controls (9). The reduction in HRQoL was similar to that observed in other chronic diseases such as rheumatoid arthritis and lupus suggesting that it is not a 'benign' disease. This reduction in QoL has been noted in multiple domains and across all populations studied worldwide. Anxiety, depression, pain and fatigue are all increased in SS compared to healthy controls and significantly impact on the QoL (10). The loss of taste and smell that accompanies SS has a negative effect on the QoL $(11)$ as does the ocular dryness $(12,13)$. There is a significant reduction in sexual QOL (14) due to the combined effects of vaginal dryness (15) and atrophy (16). Systemic involvement including nervous system disease such as peripheral neuropathy (17) and respiratory system involvement (18) also has a negative impact on QoL.

Meta-analysis suggests an increase in cardiovascular (19) and respiratory (20) morbidity and a small excess mortality has been observed in patients with SS (21), especially in males and those with underlying lung disease (22). SS remains a chronic disease with no disease modifying or curative treatments available to date and people can accumulate morbidity over time.

SS may occur alone, when it has traditionally been referred to as primary SS, or alongside another rheumatic disease when it may present as either an overlap or secondary phenomenon. The term Secondary SS has been used where the individual presents with a pre-existing autoimmune rheumatic disease, most usually rheumatoid arthritis but sometimes scleroderma or lupus. Generally the individual has had the originator disease for 10 years or more, the genetic HLA associations and antibody profile are that of the originator condition and Ro/La antibodies are usually absent. In these cases, we have previously recommended that treatment for primary SS is added to existing treatment of the prior condition, focusing especially on the treatment of the sicca symptoms. SS may present alongside other rheumatic diseases, especially systemic lupus erythematosus (SLE), scleroderma and primary biliary cholangitis. In SS/SLE overlap there is some evidence that the condition follows a more benign course than SLE alone (23). In the overlap syndromes the conditions usually present at similar time points and the antibody pattern is more often mixed with individuals 
having Ro and/or La antibodies alongside the antibody profile of the accompanying disease. In these cases, in addition to treatment of the sicca symptoms, we recommend that the treating team consider complementary immunosuppression to address both conditions. The new ACR/EULAR criteria no longer distinguish between primary and secondary SS and we will thus address the management of SS as a single entity but comment on management of overlaps where appropriate.

\section{Current practice}

A person may offer a diverse presentation with non-specific symptoms to primary or secondary care physicians, dentists, and/or opticians. The primary presentation is most commonly oral or ocular dryness but it could also range from peripheral neuropathy to gastro symptom without stated dryness. Dryness (sicca) is common, affecting $8-34 \%$ of the adult population (24), but SS itself is a relatively rare condition. The prevalence of SS is between 0.1 and $0.4 \%$ of the adult female population (25) and this rarity makes it harder for GPs to spot when they see few people with the condition. GPs must connect together diverse symptoms that may be presented to themselves, but also dentists or opticians, before entering someone into the SS diagnostic routine.

It is particularly hard to spot in children and younger adults who may not exhibit dry eyes and dry mouth symptoms due to secretory reserve. However, the children are usually positive for SSA/Ro antibodies and have parotid swelling. A pragmatic and knowledgeable approach is required by the investigating clinician to investigate these cases further despite the lack of sicca symptoms. Once SS is suspected diagnostic investigations will include blood tests looking for Ro antibodies, hypergammaglobulinaemia, low complement and lymphopaenia. These tests can occur in primary care or specialist care. Once a person is referred to specialist care they may also undergo eye tests, ocular staining scores, and some units undertake ultrasound scans of salivary glands. Minor salivary gland biopsy is an accepted part of the 2002 American-European Consensus Criteria (AECG) for Sjögren's syndrome and the more recent ACR/EULAR classification $(26,27)$ and has been shown to have high sensitivity and specificity ( $82.4 \%$ and $86.2 \%$, respectively) (26). The ACR/EULAR classification attaches similar weight to the presence of anti-SSA (Ro) antibodies as to the biopsy and allows a confirmed diagnosis to made without a biopsy if there are sufficient supporting sicca features (27).

After a positive diagnosis, prognosis and disease activity are assessed. Some units run multidisciplinary clinics but more commonly people are seen separately by rheumatology, ophthalmology and oral medicine/dentistry.

After the initial assessments people usually start with simple topical treatments for the sicca symptoms through replacement of secretions. This might be followed by stimulatory treatment, 
such as pilocarpine, for those who require it. People with significant systemic symptoms, for example, arthralgia or arthritis may benefit from treatment with hydroxychloroquine. This is usually prescribed by rheumatology and requires regular ophthalmic monitoring. Other drugs sometimes used include methotrexate, azathioprine, mycophenolate, rituximab, steroids and occasionally other immunosuppressants. Specific treatment choice aside, it is suspected that there are longer term benefits to early systemic treatment although the evidence for this is lacking to date. Demarchi et al found that individuals on hydroxychloroquine were less likely to develop extra-glandular manifestations during follow-up supporting the principle of early systemic treatment (28). There is no evidence to date that any of the pharmacological treatments are beneficial for fatigue (9).

$\mathrm{SS}$ is a chronic condition and there may be periods where non-drug approaches are effective at addressing symptoms and increasing a person's QoL. There have been studies looking at cognitive behavioural therapy, mindfulness and physical exercise for this purpose.

Some people with SS will become pregnant and give birth. There are known to be risks of complications in pregnancy arising from certain antibodies that can be present in people with SS. These risks are small and can be assessed and ameliorated through extra care during pregnancy and after birth.

Follow-up for people treated for SS is dependent on the physicians a person sees and also local healthcare provision. A driver for follow up is that people with SS are at an 8-18 fold greater risk of lymphoma than healthy people (29). These tend to be B-cell lymphomas and follow-up could be linked to an assessment of a person's chance of lymphoma development. Some patients develop significant extra-glandular manifestation such as kidney and lung disease over time (30).

\section{Who the guideline is for}

This guideline is for:

- Rheumatologists, ophthalmologists, oral medicine specialists, dentists, opticians, optometrists and other clinicians, including general practitioners, involved in the management of people with SS

- Specialist nurses and Allied Health Professionals (AHPs) involved in the management of people with SS

- People with SS

Equality considerations

- SS predominantly affects women 
3 What the guideline will cover

\subsection{Who is the focus?}

Groups that will be covered

- Adults and children with SS affecting any domains

\subsection{Settings}

Settings that will be covered

- Primary care

- Secondary \& tertiary care

\subsection{Activities, services or aspects of care}

Key areas that will be covered

We will look at evidence in the areas below when developing the guideline, but it may not be possible to make recommendations in all the areas.

1. Assessment, diagnosis and staging

2. Prevention and treatment of sicca (dryness) of oral, ocular and other systems

3. Treatment of systemic disease

4. Complementary treatment

5. Non-pharmaceutical interventions

6. Pregnancy with Sjogren's syndrome

7. Follow-up and monitoring

8. Patient information and support

\section{Areas that will not be covered}

Treatment of lymphoma

Related guidance

- The British Society for Rheumatology 2017 guideline for the management of adults with primary Sjogren's Syndrome. (2)

- Clinical practice guidelines in 2016 for Sjögren's syndrome patients in the United States by the Sjögren's Foundation (SFF). (4)

- EULAR recommendations for the management of Sjögren's syndrome with topical and systemic therapies in 2020. (3)

\subsection{Key issues and draft questions}

While writing this scope, we have identified the following key issues and draft questions related to them. The key issues and draft questions will be used to develop more detailed review questions, which guide the systematic review of the literature.

Assessment, diagnosis and staging 
1. In people suspected of Sjogren's syndrome, what is the diagnostic accuracy of ANA (antinuclear antibody), extractable nuclear antigens (ENA) and other novel antigen testing?

2. In people suspected of Sjogren's syndrome, what is the diagnostic accuracy of ultrasound?

3. In people suspected of Sjogren's syndrome, what is the diagnostic accuracy of major and minor salivary gland biopsy?

4. In people with confirmed Sjogren's syndrome are there any measurable bio-markers that can predict progression, development of extra-glandular features or lymphoma?

5. In people with SS what other investigations should routinely be undertaken to exclude common associated conditions, for example, coeliac or thyroid disease?

\section{Prevention and treatment of sicca (dryness) in SS}

6. In people with Sjogren's syndrome who have sicca (dryness) symptoms of the eyes, what is the most clinically effective topical treatment?

7. In people with Sjogren's syndrome who have sicca (dryness) symptoms of the mouth, what is the most clinically effective topical treatment?

8. In people with Sjogren's syndrome who have sicca (dryness) symptoms outside the eyes and mouth, what is the most clinically effective topical treatment?

9. In people with Sjogren's syndrome who have sicca (dryness) symptoms, what is the most clinically effective stimulatory treatment? In people with Sjogren's syndrome what is the clinical effectiveness of fluoride, xylitol, chlorhexidine, artificial saliva, or diet to prevent the development or progression of dental caries and gum disease.

\section{Treatment of systemic disease in SS}

10. In people with Sjogren's syndrome, what is the clinical effectiveness of listed treatments in comparison to each other or to placebo for treating systemic disease?

- hydroxychloroquine

- hydroxychloroquine with leflunomide

- methotrexate

- azathioprine

- rituximab

- mycophenolate

- abatacept

- tocilizumab

- Belimumab

- Ianalumab 
- JAK and BTK inhibitors

- Novel molecules

- Iscalimab

- $\quad$ RSLV-132

11. In people with Sjogren's syndrome, is early treatment of hypergammaglobulinemia or systemic disease more effective than delayed treatment at slowing disease progression?

12. What are the recommended therapeutic options in individuals with SS overlapping with other rheumatic diseases, for example, RA, SLE, Scleroderma?

\section{Complementary treatment}

13. In people with Sjogren's syndrome, what is the clinical effectiveness of nutraceuticals in the management of the condition?

\section{Non-pharmaceutical interventions}

14. For people with Sjogren's syndrome, what cognitive therapy or behavioural change interventions are an effective treatment for fatigue and joint pain?

15. In people with Sjogren's syndrome, what type and frequency of exercise is an effective treatment for fatigue?

\section{Pregnancy with Sjogren's syndrome}

16. For pregnant people with Sjogren's syndrome, both with and without anti-Ro and/or La antibodies, is hydroxychloroquine and/or low dose aspirin effective in reducing foetus mortality and morbidity?

17. For pregnant people with Sjogren's syndrome with a foetus who has an incomplete block or hydropic changes, are fluorinated steroids and/or immunoglobulins effective in decreasing the likelihood of congenital heart block in the foetus.

\section{Follow-up and monitoring}

18. In people with Sjogren's syndrome, what is the most clinically effective long-term follow-up program and how should this be personalised?

\section{Patient information and support}

19. What age-tailored information, education and support do people with Sjogren's syndrome and their families and carers need and how can they access this?

\subsection{Main outcomes}

The main outcomes that may be considered when searching for and assessing the evidence are:

- $\quad$ morbidity

- mortality

- $\quad$ quality of life 
- development of lymphoma

- development of systemic disease

- Health utility (including education, job loss, disability etc)

The guideline is expected to be published in 2021

\section{Guideline working group constituency}

Elizabeth Price (chair) - rheumatologist

Michele Bombardieri - rheumatologist

Simon Bowman - rheumatologist

Sara Carty - rheumatologist

Coziana Ciurtin - adolescent rheumatologist

Bridget Crampton - patient representative and BSSA helpline lead

Benjamin Fisher - rheumatologist

Peter Glennon - general practitioner

Kate Hackett - occupational therapist

Genevieve Larkin - ophthalmologist

Wan-Fai Ng - rheumatologist

A.V. Ramanan - paediatric rheumatologist

Saad Rassam - oncologist

Saeeha Rauz - ophthalmologist

Nurhan Sutcliffe - rheumatologist

Anwar Tappuni - oral medicine consultant

Stephen Walsh - renal physician

Dentist: to be recruited

Optician: to be recruited

Patient representative: to be recruited

\section{References}

1. British Society for Rheumatology. Creating Clinical Guidelines: Our Protocol v.5.1 Revised on behalf of SAGWG. October 2019.

2. Price EJ, Rauz S, Tappuni AR, Sutcliffe N, Hackett KL, Barone F, et al. The British Society for Rheumatology guideline for the management of adults with primary Sjogren's Syndrome.

Rheumatology (Oxford). 2017;56(10):1828. 
3. Ramos-Casals M, Brito-Zerón $\mathrm{P}$, Bombardieri S, Bootsma H, De Vita S, Dörner $\mathrm{T}$, et al. EULAR recommendations for the management of Sjögren's syndrome with topical and systemic therapies. Annals of the Rheumatic Diseases. 2020;79(1):3-18.

4. Vivino FB, Carsons SE, Foulks G, Daniels TE, Parke A, Brennan MT, et al. New Treatment Guidelines for Sjogren's Disease. Rheumatic diseases clinics of North America. 2016;42(3):531-51.

5. Ramos-Casals M, Solans R, Rosas J, Camps MT, Gil A, Del Pino-Montes J, et al. Primary Sjogren syndrome in Spain: clinical and immunologic expression in 1010 patients. Medicine. 2008;87(4):210-9.

6. Ramos-Casals M, Brito-Zeron P, Solans R, Camps MT, Casanovas A, Sopena B, et al. Systemic involvement in primary Sjogren's syndrome evaluated by the EULAR-SS disease activity index: analysis of 921 Spanish patients (GEAS-SS Registry). Rheumatology (Oxford). 2014;53(2):321-31. 7. Argyropoulou OD, Pezoulas V, Chatzis L, Critselis E, Gandolfo S, Ferro F, et al. Cryoglobulinemic vasculitis in primary Sjögren's Syndrome: Clinical presentation, association with lymphoma and comparison with Hepatitis C-related disease. Seminars in Arthritis and Rheumatism. 2020;50(5):846-53.

8. Quartuccio L, Isola M, Baldini C, Priori R, Bartoloni E, Carubbi F, et al. Clinical and biological differences between cryoglobulinaemic and hypergammaglobulinaemic purpura in primary Sjögren's syndrome: results of a large multicentre study. Scandinavian journal of rheumatology. 2015;44(1):36-41.

9. Miyamoto ST, Valim V, Fisher BA. Health-related quality of life and costs in Sjogren's syndrome. Rheumatology (Oxford). 2019.

10. Omma A, Tecer D, Kucuksahin O, Sandikci SC, Yildiz F, Erten S. Do the European League Against Rheumatism (EULAR) Sjogren's syndrome outcome measures correlate with impaired quality of life, fatigue, anxiety and depression in primary Sjogren's syndrome? Archives of medical science : AMS. 2018;14(4):830-7.

11. Al-Ezzi MY, Pathak N, Tappuni AR, Khan KS. Primary Sjogren's syndrome impact on smell, taste, sexuality and quality of life in female patients: A systematic review and meta-analysis. Modern rheumatology. 2017;27(4):623-9.

12. Zhang $\mathrm{Y}$, Lin $\mathrm{T}$, Jiang A, Zhao N, Gong L. Vision-related quality of life and psychological status in Chinese women with Sjogren's syndrome dry eye: a case-control study. BMC women's health. 2016;16(1):75.

13. Mertzanis P, Abetz L, Rajagopalan K, Espindle D, Chalmers R, Snyder C, et al. The relative burden of dry eye in patients' lives: comparisons to a U.S. normative sample. Investigative ophthalmology \& visual science. 2005;46(1):46-50. 
14. Priori R, Minniti A, Derme M, Antonazzo B, Brancatisano F, Ghirini S, et al. Quality of Sexual Life in Women with Primary Sjogren Syndrome. The Journal of rheumatology. 2015;42(8):1427-31. 15. Maddali Bongi S, Del Rosso A, Orlandi M, Matucci-Cerinic M. Gynaecological symptoms and sexual disability in women with primary Sjogren's syndrome and sicca syndrome. Clinical and experimental rheumatology. 2013;31(5):683-90.

16. Capriello P, Barale E, Cappelli N, Lupo S, Teti G. Sjogren's syndrome: clinical, cytological, histological and colposcopic aspects in women. Clinical and experimental obstetrics \& gynecology. 1988;15(1-2):9-12.

17. Jaskolska M, Chylinska M, Masiak A, Nowicka-Sauer K, Sieminski M, Zietkiewicz M, et al. Peripheral neuropathy and health-related quality of life in patients with primary Sjogren's syndrome: a preliminary report. Rheumatology international. 2020.

18. Palm O, Garen T, Berge Enger T, Jensen JL, Lund MB, Aalokken TM, et al. Clinical pulmonary involvement in primary Sjogren's syndrome: prevalence, quality of life and mortality--a retrospective study based on registry data. Rheumatology (Oxford). 2013;52(1):173-9.

19. Beltai A, Barnetche T, Daien C, Lukas C, Gaujoux-Viala C, Combe B, et al. Cardiovascular Morbidity and Mortality in Primary Sjogren's Syndrome: A Systematic Review and Meta-Analysis. Arthritis Care Res (Hoboken). 2020;72(1):131-9.

20. Flament T, Bigot A, Chaigne B, Henique H, Diot E, Marchand-Adam S. Pulmonary manifestations of Sjogren's syndrome. European respiratory review : an official journal of the European Respiratory Society. 2016;25(140):110-23.

21. Scherlinger M, Mertz P, Sagez F, Meyer A, Felten R, Chatelus E, et al. Worldwide trends in allcause mortality of auto-immune systemic diseases between 2001 and 2014. Autoimmunity reviews. 2020:102531.

22. Yazisiz V, Gocer M, Erbasan F, Ucar I, Aslan B, Oygen S, et al. Survival analysis of patients with Sjogren's syndrome in Turkey: a tertiary hospital-based study. Clinical rheumatology. 2020;39(1):233-41.

23. Xu D, Tian X, Zhang W, Zhang X, Liu B, Zhang F. Sjögren's syndrome-onset lupus patients have distinctive clinical manifestations and benign prognosis: A case-control study. Lupus. 2009;19(2):197-200.

24. Gayton JL. Etiology, prevalence, and treatment of dry eye disease. Clin Ophthalmol. 2009;3:405-12.

25. Bowman SJ, Ibrahim GH, Holmes G, Hamburger J, Ainsworth JR. Estimating the prevalence among Caucasian women of primary Sjogren's syndrome in two general practices in Birmingham, UK. Scandinavian journal of rheumatology. 2004;33(1):39-43. 
26. Vitali C, Bombardieri S, Jonsson R, Moutsopoulos HM, Alexander EL, Carsons SE, et al. Classification criteria for Sjögren\&\#039;s syndrome: a revised version of the European criteria proposed by the American-European Consensus Group. Annals of the Rheumatic Diseases. 2002;61(6):554.

27. Shiboski CH, Shiboski SC, Seror R, Criswell LA, Labetoulle M, Lietman TM, et al. 2016 American College of Rheumatology/European League Against Rheumatism classification criteria for primary Sjögren\&\#039;s syndrome. Annals of the Rheumatic Diseases. 2017;76(1):9.

28. Demarchi J, Papasidero S, Medina MA, Klajn D, Chaparro del Moral R, Rillo O, et al. Primary Sjögren's syndrome: Extraglandular manifestations and hydroxychloroquine therapy. Clinical rheumatology. 2017;36(11):2455-60.

29. Parisis D, Chivasso C, Perret J, Soyfoo MS, Delporte C. Current State of Knowledge on Primary Sjögren's Syndrome, an Autoimmune Exocrinopathy. J Clin Med. 2020;9(7):2299.

30. Ter Borg EJ, Kelder JC. Development of new extra-glandular manifestations or associated auto-immune diseases after establishing the diagnosis of primary Sjögren's syndrome : A long-term study of the Antonius Nieuwegein Sjögren (ANS) cohort. Rheumatology international. 2017;37(7):1153-8. 